University of Nebraska - Lincoln

DigitalCommons@University of Nebraska - Lincoln

$5-19-2006$

\title{
Studies by biointeraction chromatography of binding by phenytoin metabolites to human serum albumin
}

\author{
Corey M. Ohnmacht \\ University of Nebraska - Lincoln \\ Shirley Chen \\ University of Nebraska - Lincoln \\ Zenghan Tong \\ University of Nebraska - Lincoln \\ David S. Hage \\ University of Nebraska - Lincoln, dhage1@unl.edu
}

Follow this and additional works at: https://digitalcommons.unl.edu/chemistryhage

Part of the Chemistry Commons

Ohnmacht, Corey M.; Chen, Shirley; Tong, Zenghan; and Hage, David S., "Studies by biointeraction chromatography of binding by phenytoin metabolites to human serum albumin" (2006). David Hage Publications. 2.

https://digitalcommons.unl.edu/chemistryhage/2

This Article is brought to you for free and open access by the Published Research - Department of Chemistry at DigitalCommons@University of Nebraska - Lincoln. It has been accepted for inclusion in David Hage Publications by an authorized administrator of DigitalCommons@University of Nebraska - Lincoln. 


\title{
Studies by biointeraction chromatography of binding by phenytoin metabolites to human serum albumin
}

\author{
Corey M. Ohnmacht, Shirley Chen, Zenghan Tong and David S. Hage* \\ Department of Chemistry, University of Nebraska, Lincoln, NE 68588-0304 \\ *Corresponding author.Email: dhage@unlserve.unl.edu
}

\begin{abstract}
Biointeraction studies based on high performance affinity chromatography were used to investigate the binding of human serum albumin (HSA) to two major phenytoin metabolites: 5-(3-hydroxyphenyl)-5-phenylhydantoin $(m$-HPPH) and 5-(4-hydroxyphenyl)-5-phenylhydantoin ( $p$-HPPH). This was initially examined by conducting self-competition zonal elution experiments in which $m$-HPPH or $p$-HPPH were placed in both the mobile phase and injected sample. It was found that each metabolite had a single major binding site on HSA. Competitive zonal elution experiments using 1-tryptophan, warfarin, digitoxin, and cis-clomiphene as site-selective probes indicated that $m$-HPPH and $p$-HPPH were interacting with the indolebenzodiazepine site of HSA. The estimated association equilibrium constants for $m$-HPPH and $p$-HPPH at this site were 3.2 $( \pm 1.2) \times 10^{3}$ and $5.7( \pm 0.7) \times 10^{3} \mathrm{M}^{-1}$, respectively, at $\mathrm{pH} 7.4$ and $37^{\circ} \mathrm{C}$. Use of these metabolites as competing agents for injections of phenytoin demonstrated that $m$-HPPH and $p$-HPPH had direct competition with this drug at the indole-benzodiazepine site. However, the use of phenytoin as a competing agent indicated that this drug had additional negative allosteric interactions on the binding of these metabolites to HSA. These results agreed with previous studies on the binding of phenytoin to HSA and its effects on the interactions of HSA with site-selective probes for the indole-benzodiazepine site.
\end{abstract}

Keywords: phenytoin metabolites, human serum albumin, high performance affinity chromatography, biointeraction studies

\section{Introduction}

Phenytoin is one of the most widely-prescribed drugs for the treatment of epilepsy $[1,2]$. It has non-linear dose-dependent pharmacokinetics and is mostly excreted in bile as inactive metabolites, which are then reabsorbed from the intestinal tract and excreted in urine $[3,4]$. The primary metabolites of phenytoin are 5-(3-hydroxyphenyl)-5-phenylhydantoin ( $m$-HPPH) and 5-(4-hydroxyphenyl)-5-phenylhydantoin ( $p$ HPPH) (see Figure 1) [4].

Like other drug metabolites, $m$-HPPH and $p$-HPPH have similar structures to their parent drug, which can cause these to affect the distribution, action, and protein binding by phenytoin $[4,5]$. Because phenytoin exhibits dose-dependent pharmacokinetics, the apparent half-life of phenytoin changes with its dose and serum concentration. This occurs because phenytoin is hydroxylated in the liver by an enzymatic system that is saturable at high plasma levels of this drug, as occurs at therapeutic concentrations [6]. As a result, small increases in this drug's dose may cause larger-than-expected increases in phenytoin's serum concentration and apparent half-life, along with lower levels for its metabolites [6].

When phenytoin is present in blood, it is highly bound to the carrier protein human serum albumin (HSA) [3, 7]. A few reports have indicated that phenytoin interacts at the warfarinazapropazone site of HSA [8,9], while others have noted competition between phenytoin and digitoxin through the digitoxin site on HSA [10]. Recently Chen et al. have reported that phenytoin interacts at several sites on this protein [7], with association equilibrium constants at the indole-benzodiazepine and digitoxin sites of $1.04( \pm 0.05) \times 10^{4}$ and $6.5( \pm 0.6) \times 10^{3}$ $\mathrm{M}^{-1}$ at $\mathrm{pH} 7.4$ and $37^{\circ} \mathrm{C}$; interactions involving allosteric effects and/or direct binding by phenytoin at the warfarin-azapropazone and tamoxifen sites of HSA were also noted.

Besides phenytoin, HSA is known to bind to a wide range of other drugs and endogenous agents [9, 11, 12]. Although several studies have examined the binding of phenytoin to HSA, there is no known work that has examined the binding of 


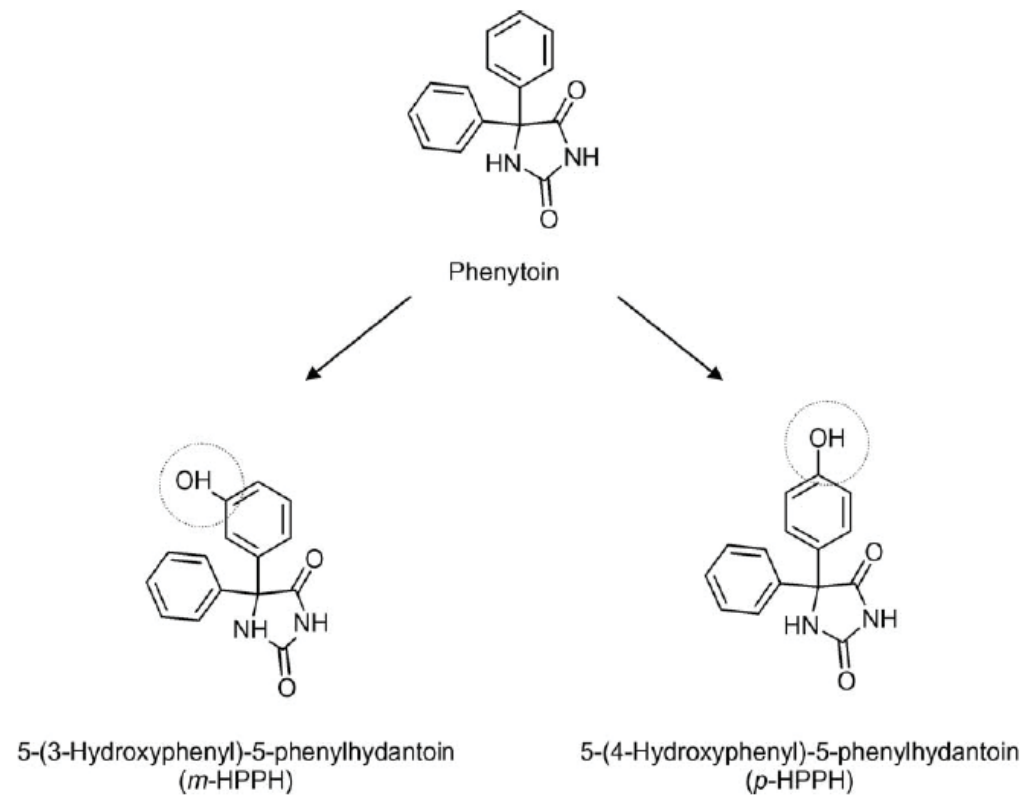

Figure 1. Structures of phenytoin and its metabolites, $m$-HPPH and $p$-HPPH.

HSA to the phenytoin metabolites $m$-HPPH and $p$-HPPH. This is of interest because $60-80 \%$ of phenytoin is metabolized into these forms [13]. The goal of this work is to study the binding of these metabolites to HSA by using high performance affinity chromatography and columns containing immobilized HSA. Numerous reports using HPLC-based HSA columns have reported drug-binding properties that show good agreement with those seen for soluble HSA [7, 14, 15]; this includes the recent work performed with phenytoin in Reference [7].

In this current study, self-competition studies with $m$ HPPH and $p$-HPPH will first be used to determine the number of binding regions and association equilibrium constants for these metabolites with HSA. The location of these binding regions will be identified through studies using warfarin, 1-tryptophan, digitoxin, and cis-clomiphene as site-selective probes [11, 16-19]. The competition of these phenytoin metabolites with phenytoin will also be considered. This should result in a more complete picture of the binding of $m-\mathrm{HPPH}$ and $p-\mathrm{HPPH}$ to HSA and of the relationship of this binding to that of phenytoin and HSA.

\section{Theory}

\subsection{General model and expressions}

The binding of $m$-HPPH and $p$-HPPH to HSA was studied by using the chromatographic method of zonal elution [16], [18], [19], [20], [21] and [22]. In this technique, a competing agent $(I)$ is placed in the mobile phase at a known concentration and continuously applied to an affinity column containing an immobilized ligand $(L)$, such as HSA. Small amounts of an analyte $(A)$ are then injected onto this system and the retention time of the analyte is determined. If $I$ and $A$ compete for a single binding site on $L$, the following reactions and equilibrium expressions can be used to describe the competition that takes place between these agents in the column.

$$
\begin{aligned}
& A+L \stackrel{K_{A L}}{\rightleftharpoons} A-L \\
& I+L \stackrel{K_{I L}}{\rightleftharpoons} I-L \\
& K_{A L}=\frac{\{A-L\}}{[A]\{L\}} \\
& K_{I L}=\frac{\{I-L\}}{[I]\{L\}}
\end{aligned}
$$

In these equations, $K_{A L}$ is the association equilibrium constant for the binding of $A$ to $L$, and $K_{I L}$ the association equilibrium constant for the binding of $I$ to $L$ at the site of competition between $A$ and $I$ [16]. The symbol [ ] represents the molar concentration of a solute in the mobile phase, while \{\} represents a surface concentration.

Equation (5) has been previously derived for the above system to describe the retention of $A$ as the value of $[I]$ is varied [16].

$\frac{1}{k}=\frac{K_{I L} V_{M}[I]}{K_{A L} m_{L}}+\frac{V_{M}}{K_{A L} m_{L}}$

In this equation, $k$ is the retention factor for the injected solute $A, V_{M}$ the column void volume, and $m_{L}$ the moles of binding sites involved in the competition of $A$ with $L$. If linear elution conditions are present (i.e., the retention factor for $A$ is independent of the amount of injected $A$ ), Equation (5) predicts that a system with direct competition at a single site will give a plot for $1 / k$ versus $[I]$ that is linear. From the ratio of the slope to the intercept of this plot it is possible to obtain $K_{I L}$, the association equilibrium constant for $I$ at its site of competition with $A$. It is also possible to obtain $K_{A L}$ from the intercept if independent values are available for $m_{L}$ and $V_{M}$ [16]. 

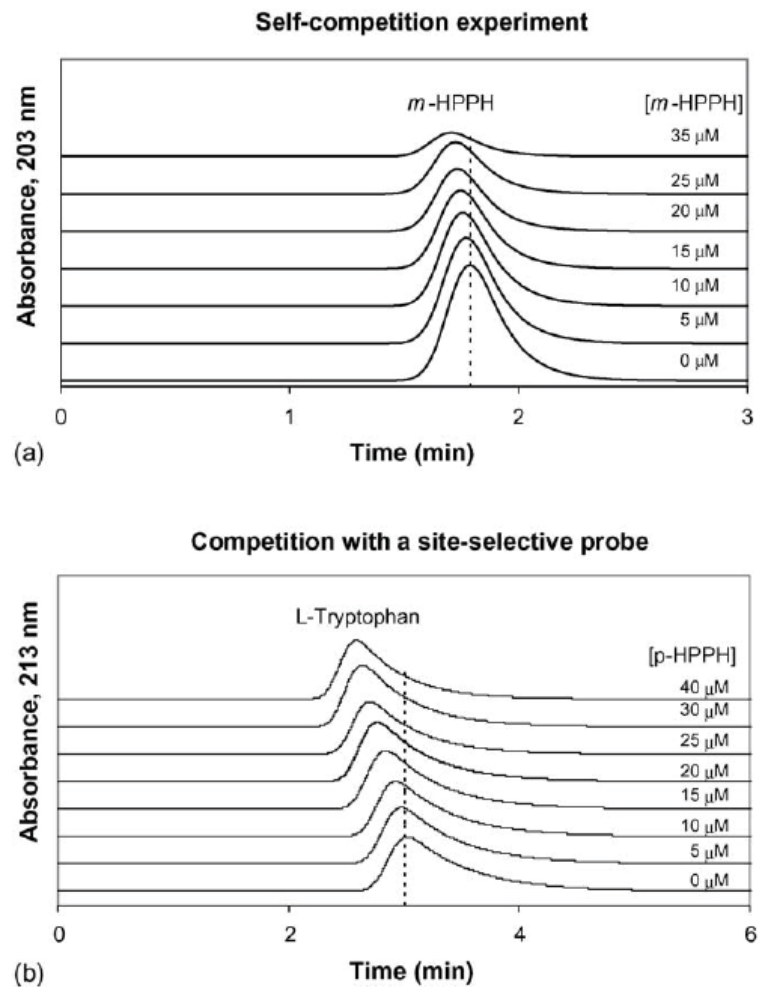

Figure 2. Typical chromatograms obtained in: (a) self-competition studies, as illustrated with data for $m-\mathrm{HPPH}$; and (b) competition studies with a site-selective probe, as represented by injections of 1-tryptophan in the presence of mobile phases containing various concentrations of $p-\mathrm{HPPH}$. The experimental conditions are given in Section 3.

\subsection{Self-competition studies}

A special case arises for the system in Eqs. (1), (2), (3) and (4) when $A$ and $I$ are the same compound. An example of such an experiment is shown in Figure 2(a). In this situation Equation (5) converts to the following form for a system with single site binding, where $[A]$ is the concentration of analyte placed into the mobile phase [16].

$\frac{1}{k}=\frac{V_{M}[A]}{m_{L}}+\frac{V_{M}}{K_{A L} m_{L}}$

This expression again results in a linear relationship for a plot of $1 / k$ versus $[A]$. However, the reciprocal of the slope now gives the effective concentration of binding sites in the column $\left(m_{L} / V_{M}\right)$, and the ratio of the slope to the intercept provides $K_{A L}$. This makes such an experiment valuable in determining both the association equilibrium constant for an analyte with $L$ and the amount of ligand sites present for this analyte in an affinity column $[7,11,12,16]$.

\subsection{Direct competition with site-selective probes}

Another advantage of zonal elution is it can be used to characterize site-specific equilibrium constants for an analyte that has multiple binding sites on a ligand. For instance, this has been used in recent work with phenytoin to show that this drug has at least two binding sites on HSA, as demonstrated by its competi- tion with the site-selective probes 1-tryptophan and digitoxin [11, 15]. This type of experiment is illustrated in Figure 2(b).

The simplest case for this type of experiment is one in which a 1:1 interaction occurs between the injected analyte $(A)$ and competing agent $(I)$ on the immobilized ligand. As demonstrated previously $[7,19]$, this situation is described by Equation (5) even if $I$ (but not $A$ ) has multiple binding regions on the ligand. In this case, the value of $K_{I L}$ that is obtained from a plot of $1 / k$ versus [ $I]$ gives the association equilibrium constant for $I$ at its site of competition of $A$. If $A$ is known to bind to a given region on the ligand, this allows the competition of $I$ at that site to also be examined and quantitated. By using different probe compounds as the injected analyte, each with a different binding region on the ligand, the interactions of $I$ at other sites on the same ligand can be examined in the same manner $[4,11,12,16,18,19]$.

\subsection{Detection of allosteric interactions}

It is important to note that a linear response to Equation (5) will be obtained only if there is direct competition between $A$ and $I$ at a single type of binding site on $L$. If this is not the case, then deviations from this linear response will be observed. One situation in which deviations can occur is if negative or positive allosteric effects are present. For instance, an allosteric effect can occur if the ligand has two distinct sites for $A$ and $I$ that affect one another.

If $A$ or $I$ is in the presence of $L$ alone, then the association equilibrium constants that will be observed for these solutes will be $K_{A L}$ and $K_{I L}$, respectively, as represented by the reactions in Equations (1) and (2). However, if both $A$ and $I$ are present and their sites are allosterically linked, then the binding of $A$ or $I$ to $L$ may cause a change in binding of the other agent at its respective site on the same ligand and result in a change in their association equilibrium constants [16, 20]. For a positive allosteric effect, this gives a non-linear response for a plot of $1 / k$ versus $[I]$, where $k$ increases with $[I]$. For a negative allosteric effect, the result is also a non-linear plot for $1 /$ $k$ versus [I], but with $k$ now decreasing as $[I]$ increases. These trends make it relatively easy to use affinity columns to qualitatively detect the presence of allosteric effects during soluteligand binding $[7,16]$. In some situations, quantitative information on such effects can also be obtained [23].

\section{Experimental}

\subsection{Reagents}

The phenytoin ( $99 \%$ pure), $m$-HPPH ( $>98 \%$ pure), $p$ HPPH ( $>98 \%$ pure), 1-tryptophan ( $>98 \%$ pure), $\beta$-cyclodextrin $(\beta-\mathrm{CD},>98 \%$ pure), digitoxin ( $97 \%$ pure) and HSA $(99 \%$ pure, essentially fatty acid free) were from Sigma (St. Louis, MO, USA). HPLC-grade dimethylsulfoxide ( $>99 \%$ pure) was from Alfa Aesar (Ward Hill, MA, USA). The cis-clomiphene was supplied by the Marion Merrell Dow Research Institute (Cincinnati, OH). The $R$-warfarin ( $>99 \%$ pure) was from Ultrafine (Manchester, England). Nucleosil Si-300 (7 $\mu \mathrm{m}$ parti- 
Ohnmacht, Chen, Tong, \& Hage in Journal of Chromatography B 836 (2006)

cle size, $300 \AA$ pore size) was obtained from Macherey-Nagel (Duren, Germany). Reagents for the bicinchoninic acid (BCA) protein assay were from Pierce (Rockford, IL, USA). Other chemicals were reagent-grade or better. All aqueous solutions were prepared using water purified by a Nanopure system (Barnstead, Dubuque, IA, USA).

\subsection{Apparatus}

The chromatographic system consisted of a Jasco PU980 pump (Easton, MD, USA), a ThermoSeparations AS3000 autosampler (Riviera Beach, FL, USA), and a Milton Roy SM3100 UV-vis absorbance detector (Riviera Beach, FL, USA). The columns were maintained at $37^{\circ} \mathrm{C}$ with a water jacket from Alltech (Deerfield, IL, USA) and water circulator from Brinkmann (Westbury, NY, USA). Chromatographic data were collected using an in-house program written in Labview 5.1 (National Instruments, Austin, TX, USA). Retention times were measured using PeakFit 4.12 (Jandel Scientific Software, San Rafael, CA, USA). All columns were downward slurrypacked with an HPLC column slurry packer from Alltech.

\subsection{Methods}

\subsubsection{Column preparation}

The immobilized HSA support was prepared as described previously [24]. In this technique, Nucleosil Si-300 was first converted to diol-bonded silica [25]. This gave $306( \pm 3) \mu \mathrm{mol}$ diol groups $( \pm 1$ S.D.) per gram of silica, as determined in triplicate by an iodometric capillary electrophoresis assay [26]. HSA was immobilized onto a portion of this support by the Schiff base method in the following manner. The diol-bonded silica (about $1 \mathrm{~g}$ being required per column) was placed in $10 \mathrm{~mL}$ of a 90:10 mixture of acetic acid and water along with a mass of sodium periodate equal to the mass of diol silica (i.e., $1 \mathrm{~g}$ sodium periodate per $1 \mathrm{~g}$ diol-bonded silica). This mixture was shaken for $2 \mathrm{~h}$ at room temperature using a wrist action shaker, followed by centrifugation and washing of the resulting aldehyde-activated support several times with deionized water. Next, $10 \mathrm{~mL}$ of $\mathrm{pH}$ 6.0, $0.1 \mathrm{M}$ phosphate buffer was used to resuspend this support. This mixture was degassed under vacuum for $15 \mathrm{~min}$ and combined with $50 \mathrm{mg}$ HSA and $25 \mathrm{mg}$ sodium cyanoborohydride per gram of silica. The resulting mixture was kept in suspension by using a rotary stirrer and allowed to react at $4{ }^{\circ} \mathrm{C}$ for five days.

After immobilization, the resulting HSA support was washed three times with $\mathrm{pH} 8.0,0.1 \mathrm{M}$ phosphate buffer. Any remaining aldehyde groups on the support were reduced by adding $17 \mathrm{mg}$ sodium borohydride per gram of silica, which was added slowly in three portions. This new mixture was placed on a wrist action shaker for $2 \mathrm{~h}$ at room temperature. The silica was then washed three times with $\mathrm{pH} 8.0,0.1 \mathrm{M}$ phosphate buffer containing $0.5 \mathrm{M}$ sodium chloride, followed by several washes with $\mathrm{pH} 7.4,0.067 \mathrm{M}$ phosphate buffer. The final support was stored in $\mathrm{pH}$ 7.4, $0.067 \mathrm{M}$ phosphate buffer at $4{ }^{\circ} \mathrm{C}$ until use. A control support was prepared in a similar manner by taking diol-bonded silica through the entire immobilization procedure but with no HSA being added.
The HSA and control supports were downward slurrypacked at $4000 \mathrm{psi}(28 \mathrm{MPa})$ into separate $4.5 \mathrm{~cm} \times 4.6 \mathrm{~mm}$ i.d. stainless steel columns using $\mathrm{pH} 7.4,0.067 \mathrm{M}$ potassium phosphate buffer as the packing solution. A portion of each remaining support was washed three times with deionized water, dried in a vacuum oven, and assayed in triplicate by the BCA method for its protein content, using HSA as the standard and the control support as the blank [27]. This measurement gave an estimated protein content for the HSA support of $28( \pm 2) \mathrm{mg}$ HSA per gram of silica.

Both the HSA and control columns were used within four months of preparation and over approximately 270 chromatographic runs. The stability of the HSA column was checked periodically by monitoring the retention factors for $m-\mathrm{HPPH}$ and $p$-HPPH in $\mathrm{pH} 7.4,0.067 \mathrm{M}$ phosphate buffer at $37^{\circ} \mathrm{C}$. Only a $13-38 \%$ decrease in these retention factors was noted during the entire course of this study. Similar experiments demonstrated that there was no significant change in the column void time (i.e., $<2 \%$ ) during these studies.

\subsubsection{Chromatographic studies}

All chromatographic experiments in this report were performed at $37^{\circ} \mathrm{C}$ and $1 \mathrm{~mL} / \mathrm{min}$, unless otherwise noted. No measurable changes in retention factors were seen when using lower flow rates (i.e., down to $0.3 \mathrm{~mL} / \mathrm{min}$ ), indicating that the given flow rate conditions were suitable for equilibrium constant measurements [7]. The typical backpressures for the HSA and control column under these conditions were 520-670 psi (4-5 MPa). All sample injections were made with a $5 \mu \mathrm{L}$ sample loop unless otherwise indicated. The detection wavelengths were as follows: $p$-HPPH and $m$-HPPH, $203 \mathrm{~nm}$; phenytoin, $205 \mathrm{~nm}$; 1-tryptophan, $213 \mathrm{~nm}$; $R$-warfarin, $308 \mathrm{~nm}$, digitoxin, $205 \mathrm{~nm}$; dimethylsulfoxide, $210 \mathrm{~nm}$; and cis-clomiphene, $204 \mathrm{~nm}$. Retention times were determined by using the first statistical moment of each chromatographic peak.

The column void time was determined by injecting dimethylsulfoxide as a non-retained solute. Similar experiments were used to correct for any non-specific binding by injecting the analytes on the control column. The retention factors measured for each analyte on the control column were then subtracted from those measured on the HSA column under the same set of experimental conditions. This correction for non-specific binding was $3-28 \%$ of the total retention measured on the HSA column for a given analyte. All retention times were also corrected for the time required for solutes to pass through the extra-column volume of the system, as determined by making injections of dimethylsulfoxide when no column was present.

All samples and mobile phases were prepared using $\mathrm{pH}$ 7.4, $0.067 \mathrm{M}$ phosphate buffer, with 2-4 replicates of all samples being injected under each set of conditions. The selfcompetition zonal elution studies with $m-\mathrm{HPPH}$ and $p-\mathrm{HPPH}$ were performed by applying solutions containing 0 to $40 \mu \mathrm{M}$ $m$-HPPH or $p$-HPPH to the HSA and control columns while injecting $50 \mu \mathrm{M}$ solutions of these same analytes. Zonal elution studies investigating the competition of phenytoin with 
its metabolites were first performed by making injections of $50 \mu \mathrm{M}$ phenytoin in the presence of $0-40 \mu \mathrm{M} m$-HPPH or $p$ $\mathrm{HPPH}$ in the mobile phase. The roles of these analytes were then reversed by placing $0-35 \mu \mathrm{M}$ phenytoin in the mobile phase while injecting $30-35 \mu \mathrm{M} m$-HPPH or $p$-HPPH. Zonal elution studies examining the competition between 1-tryptophan or $R$-warfarin with $m$-HPPH and $p$-HPPH were performed in a similar manner, using injections of $10-100 \mu \mathrm{M} 1-$ tryptophan or $20 \mu \mathrm{M} R$-warfarin as the sample.

The next set of experiments examined the use of digitoxin and cis-clomiphene as injected probes in the presence of $m$ HPPH or $p-\mathrm{HPPH}$ in the mobile phase. This was accomplished by using $\beta-C D$ in the mobile phase to aid in the solubility of digitoxin and cis-clomiphene [7, 11], as used in previous work with these probes on other HSA columns $[11,18]$. For these experiments, mobile phases containing $0-30 \mu \mathrm{M} m-\mathrm{HPPH}$ or $p$ HPPH were prepared in solutions of $2 \mathrm{mM}$ or $0.88 \mathrm{mM} \beta-\mathrm{CD}$, respectively; $\mathrm{pH} 7.4,0.067 \mathrm{M}$ phosphate buffer samples containing $5 \mu \mathrm{M}$ digitoxin or cis-clomiphene in each of these respective mobile phases were also prepared. These samples were applied using an injection volume of $20 \mu \mathrm{L}$ and a flow rate of $0.5 \mathrm{~mL} /$ min or $2 \mathrm{~mL} / \mathrm{min}$ for digitoxin and cis-clomiphene, respectively.

To ensure that linear elution conditions were present during this work, various concentrations (range, 5-100 $\mu \mathrm{M}$ ) for each analyte were injected onto both the HSA and control column in $\mathrm{pH}$ 7.4, $0.067 \mathrm{M}$ phosphate buffer while their resulting retention times were measured. No significant shifts in these retention factors (i.e., random variations of less than $3 \%$ ) were noted over this range of concentrations. Solutions containing 1-tryptophan were used on the same day as their preparation. All other samples were used within two months of preparation and stored at $4{ }^{\circ} \mathrm{C}$ in $\mathrm{pH} 7.4,0.067 \mathrm{M}$ phosphate buffer. Each solution was sonicated and degassed under vacuum for at least 15 min prior to use in chromatographic experiments.

\section{Results and discussion}

\subsection{Self-competition studies for $m-H P P H$ and $p$-HPPH}

The first set of experiments in this study used zonal elution to examine the self-competition of $m-\mathrm{HPPH}$ and $p$-HPPH on HSA. This was performed by injecting each of these phenytoin metabolites into the presence of a known concentration of the same metabolite in the mobile phase. An example of such a study is given in Figure 2(a), and the results are summarized in Table 1. Although the retention factors measured in these studies were relatively low (range, 1.22-2.17), the precision of these retention factors $( \pm 0.5-1.4 \%$, one relative standard deviation) was more than sufficient to allow the shifts in the $m$-HPPH and $p$-HPPH peaks to be observed and determined in a reproducible fashion. The retention data obtained in these experiments were analyzed according to a 1:1 direct competition model by using Equation (6). The graphs this produced are given in Figure 3 . It was found that plots of $1 / k$ versus $[A]$ were linear for both metabolites, with correlation coefficients for $m$-HPPH and $p$-HPPH of $0.9971(n=7)$ and $0.9942(n=7)$, respectively. The best-fit intercepts for both metabolites were within one standard
Table 1

Retention factors obtained in self-competition studies for $m$-HPPH and $p$-HPPH on an immobilized HSA column ${ }^{\mathrm{a}}$

\begin{tabular}{llll}
\hline$[m-\mathrm{HPPH}](\boldsymbol{\mu M})$ & $k_{(m-\mathrm{HPPH})}$ & {$[p-\mathrm{HPPH}](\mu \mathrm{M})$} & $k_{(p-\mathrm{HPPH})}$ \\
\hline 0 & $1.39( \pm 0.01)$ & 0 & $2.17( \pm 0.03)$ \\
5 & $1.36( \pm 0.01)$ & 5 & $2.10( \pm 0.01)$ \\
10 & $1.34( \pm 0.01)$ & 10 & $2.07( \pm 0.01)$ \\
15 & $1.31( \pm 0.01)$ & 15 & $2.03( \pm 0.02)$ \\
20 & $1.29( \pm 0.01)$ & 20 & $2.00( \pm 0.01)$ \\
25 & $1.27( \pm 0.01)$ & 25 & $1.97( \pm 0.01)$ \\
35 & $1.22( \pm 0.01)$ & 30 & $1.94( \pm 0.02)$ \\
& & 40 & $1.91( \pm 0.02)$
\end{tabular}

a The values in parentheses represent a range of \pm 1 S.D. All of these measurements were performed at $37^{\circ} \mathrm{C}$ in $\mathrm{pH} 7.4,0.067 \mathrm{M}$ phosphate buffer.

deviation of the true intercepts, giving differences of only 0.2 and $1.1 \%$ in these values for $m$-HPPH and $p$-HPPH.

The linearity of the plots in Figure 3 and the agreement between the actual and best-fit intercepts of these plots supports a model in which both phenytoin metabolites are interacting at only one major site on HSA. From the slope/intercept ratios of these plots, it was possible to obtain the association equilibrium constants for $m-\mathrm{HPPH}$ and $p$-HPPH with HSA. This gave estimated values of $4.0( \pm 0.1) \times 10^{3} \mathrm{M}^{-1}$ and $5.2( \pm 0.3) \times 10^{3} \mathrm{M}^{-1}$, respectively, at $\mathrm{pH} 7.4$ and $37^{\circ} \mathrm{C}$. The $23 \%$ difference in these constants was statistically significant and indicated that the different positions of the hydroxyl groups on $m$-HPPH and $p$-HPPH had a measurable effect on the binding of these metabolites to HSA. It is also important
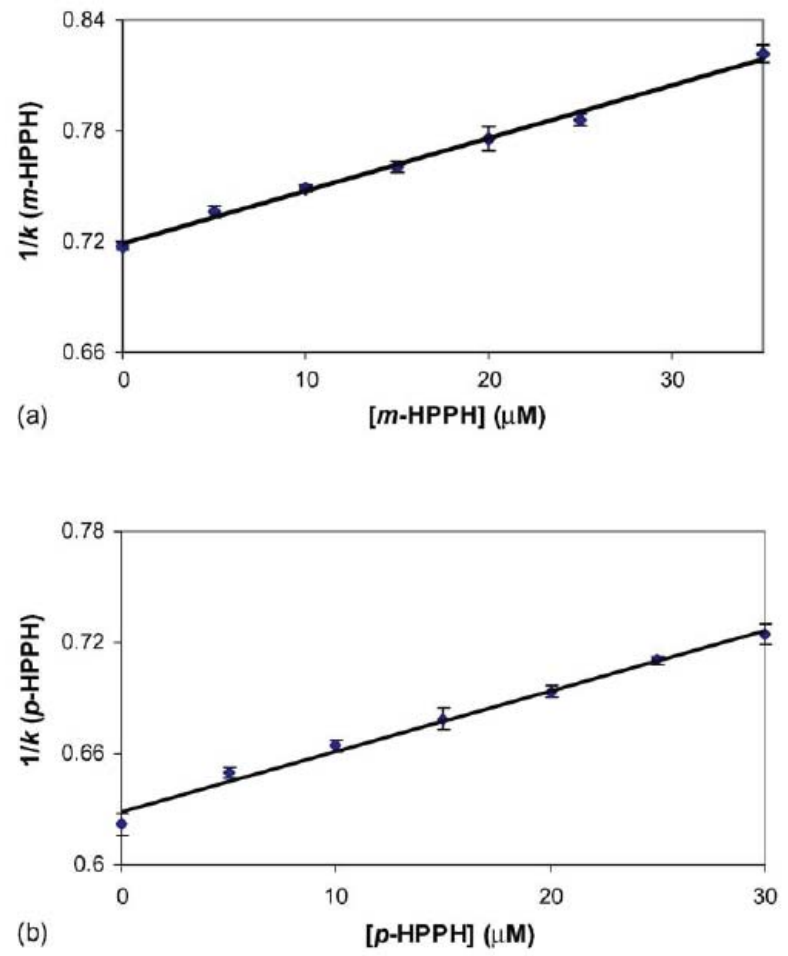

Figure 3. Results of self-competition zonal elution studies for: (a) $m-\mathrm{HPPH}$; and (b) $p$-HPPH, as plotted according to Equation (6). The experimental conditions are given in the text. The best-fit lines were: (a) $y=0.00256( \pm 0.000$ 10) $x+0.7188( \pm 0.0019)$; and (b) $y=0.0033( \pm 0.0002) x+0.6285( \pm 0.0029)$, with correlation coefficients of $0.9971(n=7)$ and $0.9942(n=7)$, respectively. The error bars represent a range of \pm 1 S.D. 
Ohnmacht, Chen, Tong, \& Hage in Journal of Chromatography B 836 (2006)

to note that these values are significantly less (i.e., $20-68 \%$ lower) than the association equilibrium constants that have been measured on similar columns for phenytoin [7].

As noted in recent theoretical studies [28,29], the observed peaks in self-competition studies are actually due to analyte in the mobile phase that has been displaced by the sample rather than due to the injected analyte, which elutes slightly later as a normally unobservable mass peak. This means the retention factors in Table 1 are lower than those for the true peak of interest (i.e., the mass peak), which would give high estimates for association equilibrium constants determined according to Equation (6). However, it is possible to determine the maximum size of this error based on the estimated separation factor $(\alpha)$ between the mass peak and displacement peak, as described in Reference [28]. Under the conditions used in this study this gave a maximum difference of only $7-12 \%$ in the desired and observed retention factors. This same issue was not a problem in later studies using site-selective probes or phenytoin as competing agents (see Sections 4.2 and 4.3) since in these experiments the injected analyte was different from the competing agent and had a mass peak that could be observed directly.

\subsection{Competition of phenytoin metabolites with site-selective probes for HSA}

The next group of studies sought to determine the location of the major binding regions for $m$-HPPH and $p$-HPPH on HSA. This was accomplished by examining the competition of these phenytoin metabolites with l-tryptophan and $R$ warfarin, which are often used as probes for the indole-benzodiazepine and warfarin-azapropazone sites of HSA (i.e., Sudlow sites I and II) $[15,17,18]$. The data obtained in these experiments are summarized in Table 2. The first series of experiments used injections of 1-tryptophan in the presence of $m$-HPPH or $p$-HPPH as a mobile phase additive, as illustrated earlier in Figure 2(b). A plot of $1 / k$ versus $[I]$ was then made for each phenytoin metabolite. According to Equation (5), this type of plot should yield a straight line if the probe and mobile phase additive are competing at a single type of site on the immobilized ligand.

The plots obtained when using 1-tryptophan as a site-selective probe are shown in Figure 4, where both phenytoin metabolites gave linear relationships when their data were analyzed according to Equation (5). The correlation coefficients of the graphs for $m$-HPPH and $p$-HPPH were $0.9956(n=6)$ and $0.9986(n=7)$, respectively. In addition, both plots gave best-fit intercepts that agreed within one standard deviation with the actual intercepts, with values that differed by only 0.3 and $0.4 \%$. This type of fit indicated that both metabolites had direct 1:1 competition with l-tryptophan. This led to the conclusion that the indole-benzodiazepine site (i.e., Sudlow site II) was the major binding region on HSA for both $m$-HPPH and $p$-HPPH.

From the intercept and slope of the plots in Figure 4, it was possible to obtain additional estimates of the association equilibrium constants for $m$-HPPH and $p$-HPPH with HSA. This gave association equilibrium constants of $2.3( \pm 0.1) \times 10^{3} \mathrm{M}^{-1}$

\begin{tabular}{lccc} 
Table 2 & & \\
Retention factors obtained in competition studies on an immobilized HSA col- \\
umn for $m$-HPPH and $p$-HPPH in the presence of various site-selective probes \\
\hline [
\end{tabular}

and $6.2( \pm 0.1) \times 10^{3} \mathrm{M}^{-1}$, respectively, for these metabolites at $\mathrm{pH} 7.4$ and $37^{\circ} \mathrm{C}$. These values, which were now measured for these metabolites specifically at the indole-benzodiazepine site, are in reasonable agreement with those determined earlier from the self-competition studies. These results again indicate that $p$ HPPH has stronger binding to HSA than $m$-HPPH and that both metabolites had weaker binding to HSA than phenytoin [7].

Similar studies were conducted using $R$-warfarin as a siteselective probe for the warfarin-azapropazone site of HSA (i.e., Sudlow site I). However, in this case only small random variations $( \pm 2 \%)$ in the retention factor of the injected probe were noted as the mobile phase concentration of $m$-HPPH or $p$-HPPH was varied. This indicated that these phenytoin metabolites were not binding at the warfarin-azapropazone site. These results also indicated that these metabolites did not have any appreciable allosteric effects on the binding of $R$-warfarin to HSA. This result differs from previous studies with $R$ warfarin and phenytoin, where allosteric effects plus possible direct competition were shown to exist between these agents during their interactions with HSA [7].

Additional studies were conducted to investigate any interactions of the phenytoin metabolites at the digitoxin and tamoxifen sites of HSA. This was accomplished by making injections of digitoxin (a digitoxin site probe) or cis-clomi- 

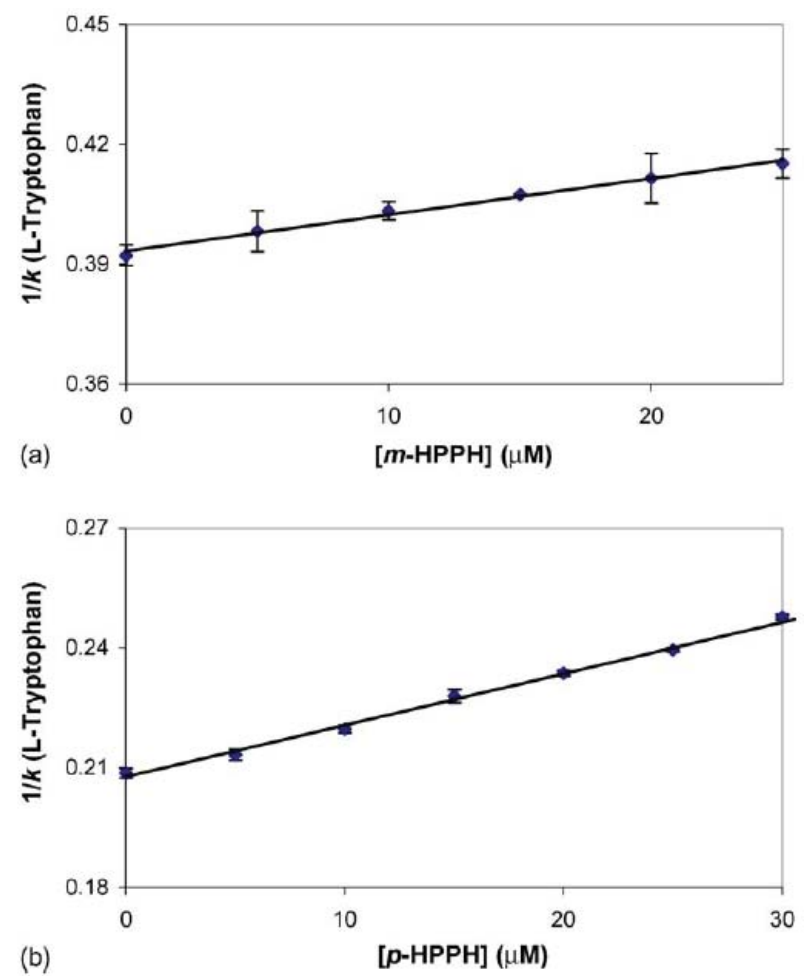

Figure 4. Results of zonal elution studies using 1-tryptophan as a site-specific probe injected in the presence of mobile phases containing known concentrations of: (a) $m$-HPPH; or (b) $p$-HPPH when analyzing the data according to Equation (5). The best-fit lines were: (a) $y=0.00091( \pm 0.00004) x+0.3933$ ( $\pm 0.0006)$; and (b) $y=0.00129( \pm 0.00003) x+0.2078( \pm 0.0006)$, with correlation coefficients of $0.9956(n=6)$ and $0.9986(n=7)$, respectively. The error bars represent a range of \pm 1 S.D.

phene (a tamoxifen site probe) in the presence of mobile phases that contained known levels of $m$-HPPH or $p$-HPPH. For both digitoxin and cis-clomiphene only small random variations $( \pm 1-4 \%)$ in their retention factors were observed as the mobile phase concentration of $m$-HPPH or $p$-HPPH was varied. The digitoxin site has been found previously to bind phenytoin [7], but the results obtained in this current study indicate that $m$-HPPH and $p$-HPPH do not have significant interactions at this site. This may be related to the greater polarity of these metabolites versus phenytoin, which would tend to decrease the importance of non-polar interactions in the binding of these compounds to HSA. Alternatively, this may result from steric effects that prevent the binding of these metabolites to the digitoxin site due to the presence of the hydroxyl groups on these compounds.

\subsection{Competition of phenytoin with $m-H P P H$ and $p-H P P H$}

A third set of experiments investigated the competition that occurs on HSA between $m-\mathrm{HPPH}$ and $p-\mathrm{HPPH}$ and their parent compound, phenytoin. This was of interest because of the differences noted earlier in this report concerning the association equilibrium constants and number of binding sites for phenytoin compared to its metabolites. The results obtained in these studies are summarized in Table 3.
Table 3

Retention factors obtained in competition studies on an immobilized HSA column for $m$-HPPH and $p$-HPPH vs. phenytoin ${ }^{\mathrm{a}}$

\begin{tabular}{llll}
\hline$[m-\mathrm{HPPH}](\mu \mathrm{M})$ & $k_{\text {(Phenytoin) }}$ & {$[p$-HPPH $](\mu \mathrm{M})$} & $k_{\text {(Phenytoin) }}$ \\
\hline 0 & $2.18( \pm 0.01)$ & 0 & $2.45( \pm 0.01)$ \\
5 & $2.15( \pm 0.01)$ & 5 & $2.43( \pm 0.06)$ \\
10 & $2.14( \pm 0.01)$ & 10 & $2.35( \pm 0.02)$ \\
15 & $2.11( \pm 0.04)$ & 15 & $2.32( \pm 0.01)$ \\
20 & $2.09( \pm 0.01)$ & 20 & $2.26( \pm 0.02)$ \\
25 & $2.07( \pm 0.01)$ & 25 & $2.23( \pm 0.01)$ \\
35 & $2.02( \pm 0.02)$ & 40 & $2.14( \pm 0.01)$ \\
& & & \\
{$[$ Phenytoin] $(\mu \mathrm{M})$} & $k_{(m-\mathrm{HPPH})}$ & {$[$ Phenytoin $](\mu \mathrm{M})$} & $k_{(p-\mathrm{HPPH})}$ \\
\hline 0 & $1.46( \pm 0.01)$ & 0 & $1.33( \pm 0.01)$ \\
4.48 & $1.43( \pm 0.01)$ & 4.48 & $1.31( \pm 0.01)$ \\
8.96 & $1.41( \pm 0.01)$ & 8.96 & $1.29( \pm 0.01)$ \\
17.92 & $1.39( \pm 0.01)$ & 17.92 & $1.28( \pm 0.01)$ \\
31.35 & $1.36( \pm 0.01)$ & 31.35 & $1.25( \pm 0.01)$ \\
\hline \multicolumn{2}{c}{ a The values in parentheses represent a range of \pm 1 S.D. All of these mea- } \\
surements were performed at $37^{\circ} \mathrm{C}$ in pH $7.4,0.067 \mathrm{M}$ phosphate buffer.
\end{tabular}

The first series of zonal elution experiments in this section were performed by injecting trace amounts of phenytoin into mobile phases that contained known concentrations of its metabolites. Figure 5 shows the plots obtained when the data were analyzed using a 1:1 direct competition model, as represented by Equation (6). These plots gave linear responses,
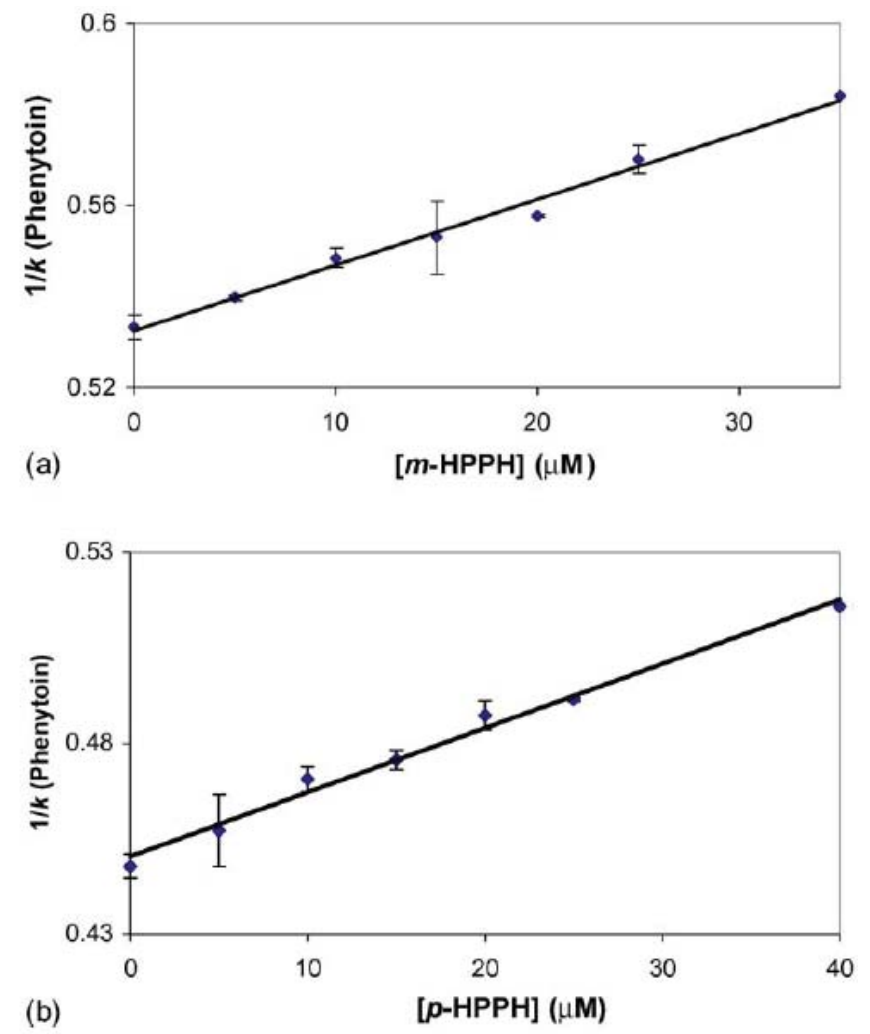

Figure 5. Results of zonal elution competition studies using phenytoin injected into mobile phases containing known concentrations of: (a) $m$-HPPH; or (b) $p$-HPPH. The best-fit lines were: (a) $y=0.00144( \pm 0.00007) x+0.5325$ ( $\pm 0.0014)$; and (b) $y=0.00169( \pm 0.00008) x+0.4501( \pm 0.0017)$, with correlation coefficients of $0.9939(n=7)$ and $0.9939(n=7)$, respectively. The error bars represent a range of \pm 1 S.D. 
Table 4

Association equilibrium constants $\left(K_{A L}\right)$ and types of binding for phenytoin and its metabolites to various regions on $\mathrm{HSA}^{\mathrm{a}}$

\begin{tabular}{|c|c|c|c|}
\hline \multirow[t]{2}{*}{ Binding region on $\mathrm{HSA}$} & \multicolumn{3}{|l|}{ Agent binding to HSA } \\
\hline & Phenytoin & $m$-HPPH & p-HPPH \\
\hline $\begin{array}{l}\text { Warfarin-azapropazone site } \\
\text { (Sudlow site I) }\end{array}$ & $\begin{array}{l}\text { Allosteric effects }+ \text { possible } \\
\text { direct binding }\end{array}$ & No binding & No binding \\
\hline $\begin{array}{l}\text { Indole-benzodiazepine site } \\
\text { (Sudow site II) }\end{array}$ & $\begin{array}{l}\text { Direct binding } \\
K_{A L}=1.04 \times 10^{4} \mathrm{M}^{-1}\end{array}$ & Direct binding $K_{A L}=3.2 \times 10^{3} \mathrm{M}^{-1}$ & Direct binding $K_{A L}=5.7 \times 10^{3} \mathrm{M}^{-1}$ \\
\hline Digitoxin site & $\begin{array}{l}\text { Direct binding } \\
K_{A L}=6.5 \times 10^{3} \mathrm{M}^{-1}\end{array}$ & No binding & No binding \\
\hline Tamoxifen site & $\begin{array}{l}\text { Allosteric effects }+ \text { possible } \\
\text { direct binding }\end{array}$ & No binding & No binding \\
\hline
\end{tabular}

${ }^{\text {a }}$ All of these measurements were performed at $37^{\circ} \mathrm{C}$ in $\mathrm{pH} 7.4,0.067 \mathrm{M}$ phosphate buffer. The results for phenytoin were obtained from Ref. [7].

as predicted for direct competition at a single site, with correlation coefficients of $0.9939(n=7)$ and $0.9939(n=7)$ for the $m$-HPPH and $p$-HPPH data, respectively. The best-fit intercepts were within one standard deviation of the true intercepts, with differences in these values of only 0.14 and $0.55 \%$ for $m$-HPPH and $p$-HPPH. It was possible from the slopes and intercepts of these plots to again obtain estimates of the association equilibrium constants for $m-\mathrm{HPPH}$ and $p$-HPPH with HSA. This gave values of $2.7( \pm 0.1) \times 10^{3} \mathrm{M}^{-1}$ and 3.8 $( \pm 0.2) \times 10^{3} \mathrm{M}^{-1}$, respectively, which agreed with the numbers calculated earlier from Figure 3 and Figure 4 .

Figure 5 indicates that both $m$-HPPH and $p$-HPPH had direct competition with phenytoin on HSA. From the experiments described in the last section, it was further determined that this competition must be occurring at the indole-benzodiazepine site. However, as also stated earlier, phenytoin is known to have additional binding regions on HSA and to have significant allosteric effects on solutes that bind to other sites on this protein [7]. The experiments in Figure 5 did not allow such effects to be seen since phenytoin was present in only a trace amount compared to its metabolites. To overcome this limitation, the reverse experiment was next performed, in which the phenytoin metabolites were injected into mobile phases that contained known concentrations of phenytoin. The results are shown in Figure 6.

When the data for the competition of $p$-HPPH with phenytoin were plotted according to the direct competition model in Equation (6), the results gave a good fit to a linear regression model, as shown in Figure 6(b), with a correlation coefficient of $0.9913(n=5)$. However, the association equilibrium constant estimated from this plot for phenytoin was $1.8( \pm$ $0.1) \times 10^{3} \mathrm{M}^{-1}$. This value is approximately an order of magnitude lower than that found in previous competition studies using a trace amount of phenytoin and l-tryptophan as a site-selective probe for the indole-benzodiazepine site [7]. As indicated in Reference [7], this decrease in binding strength for phenytoin can be explained by a negative allosteric effect between phenytoin and itself as this drug binds to other regions on HSA. This would also be expected to produce a mixed-mode interaction between phenytoin and $p-\mathrm{HPPH}$, where phenytoin can undergo either direct competition with this metabolite or cause a negative allosteric effect in the binding of $p$-HPPH to HSA.
The presence of negative allosteric effects was more clearly indicated in the results for $m$-HPPH and phenytoin in Figure 6(a). In this case, when the data were plotted according to the direct competition model in Equation (6) distinctly nonlinear behavior was noted. This behavior is consistent with a model in which the binding of phenytoin to HSA is having direct competition plus a negative allosteric effect on the binding of its metabolite to HSA. A similar effect to that seen in Figure 5 and Figure 6 has been also reported in earlier studies examining the interactions on HSA between phenytoin with 1tryptophan (i.e., the site-selective probe used in this current study for the indole-benzodiazepine site) $[7,20]$.
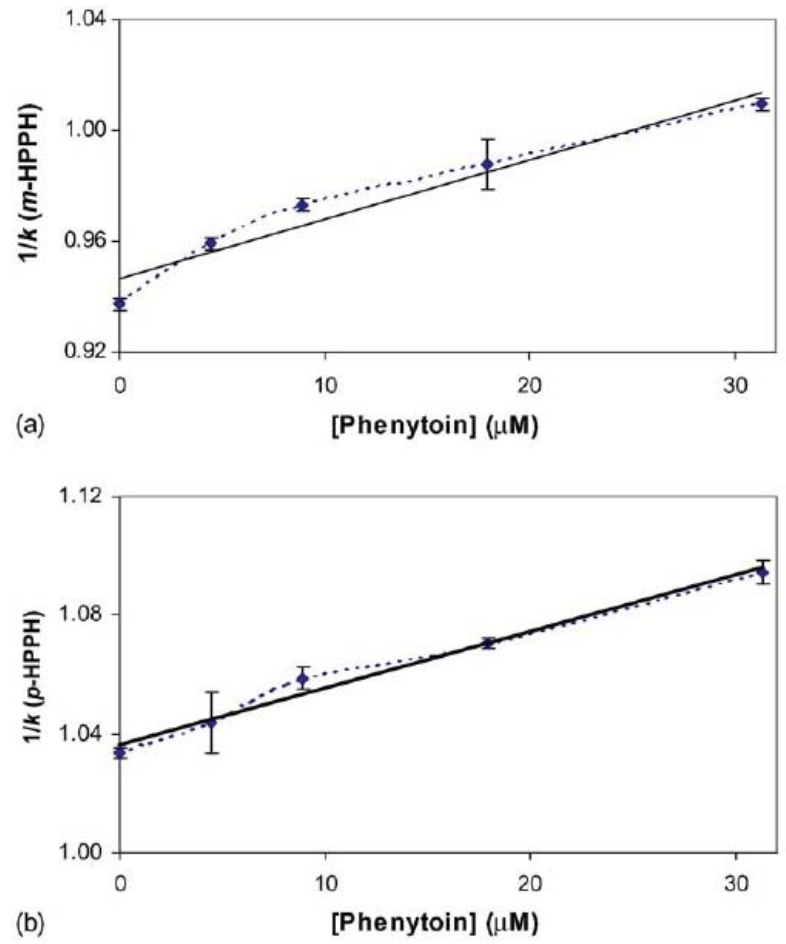

Figure 6. Results of zonal elution competition studies using: (a) $m$-HPPH; or (b) $p$-HPPH injected into the presence of mobile phases containing known concentrations of phenytoin. The solid-lines show the best-fit response when the data were analyzed according to a 1:1 direct competition model based on Equation (5). The best-fit lines in these plots were: (a) $y=0.0022( \pm 0.0003) x$ $+0.9709( \pm 0.0051)$; and (b) $y=0.0019( \pm 0.0001) x+1.0363( \pm 0.0024)$ with correlation coefficients of $0.9709(n=5)$ and $0.9913(n=5)$, respectively. The error bars represent a range of \pm 1 S.D. 


\section{Conclusions}

This report used HPLC-based immobilized HSA columns to study binding by the phenytoin metabolites $m$-HPPH and $p$ HPPH to HSA. The results are summarized in Table 4. Selfcompetition experiments indicated that these metabolites had a single major binding site on HSA. Further work with siteselective probes indicated that the indole-benzodiazepine site (i.e., Sudlow site II) was the location of this binding region. Several estimates were obtained for the association equilibrium constants of these phenytoin metabolites with HSA. These values ranged from 2.3 to $4 \times 10^{3} \mathrm{M}^{-1}$ (best estimate, $\left.3.2( \pm 1.2) \times 10^{3} \mathrm{M}^{-1}\right)$ for $m$-HPPH and $5.2-6.2 \times 10^{3} \mathrm{M}^{-1}$ (best estimate, $\left.5.7( \pm 0.7) \times 10^{3} \mathrm{M}^{-1}\right)$ for $p$-HPPH at $\mathrm{pH} 7.4$ and $37^{\circ} \mathrm{C}$. For each of these estimates, $p$-HPPH had an association equilibrium constant that was 1.3-2.7 fold higher than that for $m$-HPPH. This indicated the relative importance of the hydroxyl group position of these compounds in determining their overall binding strength to HSA.

Both $m-\mathrm{HPPH}$ and $p$-HPPH where found to have direct competition with their parent compound, phenytoin, at the indole-benzodiazepine site of HSA. However, experiments using phenytoin as a competing agent indicated that this drug also had negative allosteric interactions on the binding of these metabolites to HSA. These results agree with previous studies on the binding of phenytoin to HSA and its effects on the interactions of HSA with site-selective probes for the indole-benzodiazepine site [7] and [20]. The results of this report also demonstrate the value of using HPLC-based HSA columns in the study of drug/metabolite competition. In addition, these results indicate that significant differences can occur in both the binding strength and binding regions for drugs and their metabolites on HSA.

\section{Acknowledgment}

This work was supported under grant R01 GM044931 from the National Institutes of Health.

\section{References}

[1] G. F. Ayala and D. Johnston, Epilepsia 18 (1977), p. 299.

[2] T. J. Putman and H. H. Merritt, Science 85 (1937), p. 525.

[3] H. Kodama, Y. Kodama, S. Shinozawa, R. Kanemaru, K. Todaka and Y. Mitsuyama, J. Clin. Pharm. Therapeut. 23 (1998), p. 361.

[4] S. Hara, J. Hagiwara, M. Fukuzawa, N. Ono and T. Kruoda, Anal. Sci. 15 (1999), p. 371.

[5] C. J. Bowmer and E.W. Lindup, Biochem. Pharmacol. 27 (1978), p. 937.

[6] T. U. S. P. Convention Drug Information for the Health Care Professional, 17 ed., Rand McNally, Tauton, 1997.

[7] J. Chen, C. Ohnmacht and D. S. Hage, J. Chromatogr. B 809 (2004), p. 137.

[8] I. Sjoeholm, B. Ekman, A. Kober, I. Ljungstedt-Pahlman, B. Seiving and T. Sjodin, Mol. Pharmacol. 16 (1979), p. 767.

[9] A. Kober, Y. Olsson and I. Sjoholm, Mol. Pharmacol. 18 (1980), p. 237.

[10] A. Dasgupta, A. E. Vega, A. Wells and P. Datta, Ther. Drug. Monit. 21 (1999), p. 625.

[11] D. S. Hage and A. Sengupta, J. Chromatogr. B 724 (1998), p. 91.

[12] D. S. Hage and A. Sengupta, Anal. Chem. 70 (1998), p. 4602.

[13] S. J. Soldin, E. Wang, Z. Verjee and R. J. Elin, Arch. Pathol. Lab Med. 127 (2003), p. 1623.

[14] D. S. Hage and S. A. Tweed, J. Chromatogr. B 699 (1997), p. 499.

[15] J. Yang and D. S. Hage, J. Chromatogr. 645 (1993), p. 241.

[16] D. S. Hage, J. Chromatogr. B 768 (2002), p. 3.

[17] B. Loun and D. S. Hage, Anal. Chem. 68 (1996), p. 1218.

[18] D. S. Hage and A. Sengupta, Anal. Chem. 70 (1998), p. 4602.

[19] H. S. Kim and D. S. Hage, J. Chromatogr. B 816 (2005), p. 57.

[20] C. Andre, Y. Jacquot, T. T. Truong, M. Thomassin, J. F. Robert and Y. C. Guillaume, J. Chromatogr. B 796 (2003), p. 267.

[21] C. Bertucci, M. Bartolini, R. Gott and V. Andrisano, J. Chromatogr. B 797 (2003), p. 111.

[22] L. Renyu, R. Pidikiti, C. Ha, C. E. Petersen, N. V. Bhagavan and R. G. Eckenhoff, J. Biol. Chem. 277 (2002), p. 363.

[23] J. Chen and D. S. Hage, Nat. Biotechnol. 22 (2004), p. 1445.

[24] P. O. Larsson, Methods Enzymol. 104 (1984), p. 212.

[25] P. F. Ruhn, S. Garver and D. S. Hage, J. Chromatogr. A 669 (1994), p. 9.

[26] A. Chattopadhyay and D. S. Hage, J. Chromatogr. A 758 (1997), p. 255.

[27] P. K. Smith, R.I. Krohn, G. T. Hermanson, A. K. Malia, F. H. Gartner, M. D. Provenzano, E. K. Fujimoto, N. M. Goeke, B. J. Olson and D. C. Klenk, Anal. Biochem. 150 (1985), p. 76.

[28] J. Samuelsson, P. Forssen, M. Stefansson and T. Fornstedt, Anal. Chem. 76 (2004), p. 953.

[29] F. Helfferich and D. L. Peterson, Science 142 (1963), p. 661. 